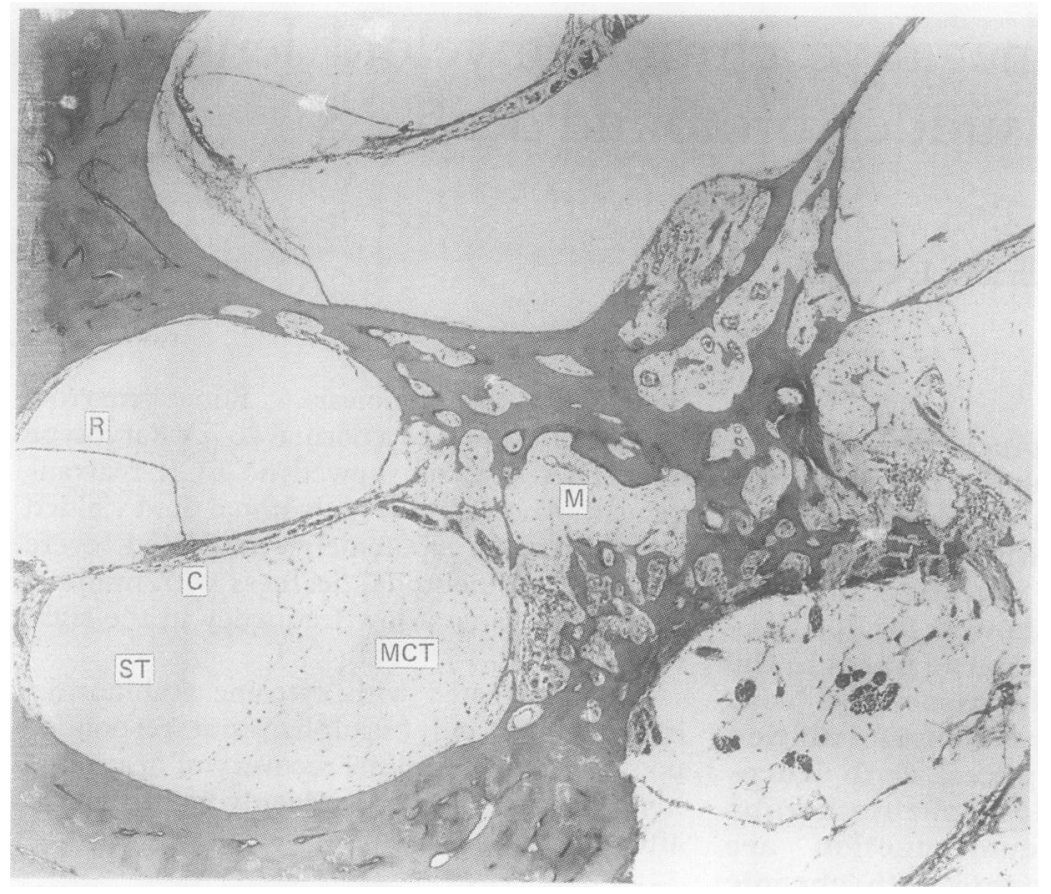

Section of right cochiea through the region of basal and midau coils. The scala tympani $(S T$ ) and modiolus $(M)$ of the basal coil contain a deposit of myxoid connective tissue (MCT). There is severe reduction of spiral ganglion cells in modiolar spaces that wouid normally contain many. The organ of Corti $(C)$ is atrophic in all places. There is moderate hydrops with some outward bulging of Reissner's membrane $(R)$

haematoxwin and eosin .

both cochlea and vestibules were severely damaged with atrophy of basilar membrane nerve fibres and spiral and vestibular ganglion cells. These changes may represent the end and vestibular structures. Such changes most frequently result from severe labyrinthine infection, usually secondary to otitis media or meningitis, but there was no clinical evidence of infection in this patient. Hyperviscosity is unlikely to lead to such profound permanent changes affecting sensory and ganglion cells or labyrinthine structures. Haemorrhage could cause widespread labyrinthine changes but these are usually irregular. There was no evidence of haemosiderin or cholesterol granuloma at post mortem to suggest this as a cause. The most likely cause seems to be leukaemic infiltration of the labyrinthine spaces involving the perilymphatic space and basilar membrane; disappearance of leukaemic cells with treatment could be followed by extensive fibrosis and bony reaction with loss of sensory cells and their nerve supply. ${ }^{6}$ The cranial nerve palsies remain unexplained.

It is difficult to distinguish between deafness due to hyperviscosity or leukaemic infiltration in patients with $\mathrm{ML}$. We advocate both leucopheresis and high dose chemotherapy capable of rapid cytoreduction, as convential chemotherapy is rarely followed by recovery. ${ }^{4}$

1 Paparella MM, Beringer NT, Oda M, Fiky FE. Otological manifestations of leukemia. Laryngoscope 1973;83: 1510-26.

2 Preston FE, Sokol RJ, Lilleyman JS, Winfield DA, Blackburn EK. Cellular hyperviscosity as a cause of nurn
i: $476-8$.

3 Thompson RB. The clinical and haematological features of chronic granulocytic leukaemia in the chronic phase. In: Shaw MT ed. Chronic granulocytic leukaemia. Eastbourne: Praeger, 1982:145-8.

4 Williams CKO, Ogan O. Chronic myeloid leukaemia associated with impairment of hearing. $\mathrm{Br} M e d$ 1985;1:1705.

5 Onwikeme KE, Sagoe AS, Bhatia PL. Sensori-neural hearing impairment in chronic granulocytic leukaemia. Trop Geog Med 1987;39:24-7.

6 Michaels L. Ear, nose and throat histopathology. London Springer Verlag, 1987.

Department of
Medicine for the
Elderly, Manchester
Royal Infirmary,
Manchester
F J Thomson
C M Cheshire
Department of
Pathological Sciences,
University of
Manchester
D E W Benbow
R F T McMahon
Correspondence to:
Dr F J Thomson, Diabetes
Unit, Hope Hospital, Eccles
Old Road, Salford M6 8HD
Accepted for publication
22 May 1991

F J Thomson, E W Benbow, R F T McMahon, C M Cheshire

\title{
Pulmonary infarction, myocardial infarction, and acute disseminated intravascular coagulation
}

\author{
Abstract \\ Pulmonary and myocardial damage are \\ frequently cited as manifestations of dis- \\ seminated intravascular coagulation \\ (DIC), but rarely as causes. Three elderly \\ cases of severe DIC due to pulmonary and \\ myocardial infarction are reported. All \\ three patients died. Necropsy showed \\ extensive pulmonary emboli in each case \\ with large pulmonary infarcts in cases 1 \\ and 2 and a ventricular aneurysm con- \\ taining thrombus in cases 2 and 3. Early \\ diagnosis and treatment of pulmonary \\ embolism requires a high degree of
}

clinical suspicion but may prevent progression to the irreversible stage of severe DIC.

Disseminated intravascular coagulation (DIC) is characterised by inappropriate activation of the coagulation system, with consumption of coagulation factors and secondary fibrinolysis. ${ }^{1}$ It is not a single disease process, but a common pathway in a large number of primary disorders. Pulmonary and myocardial damage are frequently cited as manifestations of DIC, which we believe obscures the fact they also number among its causes. 
Haematological results of the three patients confirming the diagnosis of disseminated intravascular coagulation (DIC)

\begin{tabular}{lllll}
\hline & Reference range & Case 1 & Case 2 & Case 3 \\
\hline Haemoglobin & $11 \cdot 5-15 \cdot 6 \mathrm{~g} / 1$ & $12 \cdot 4$ & $14 \cdot 5$ & $14 \cdot 8$ \\
Platelets & $150-400 \times 10^{9} / 1$ & 82 & 41 & 25 \\
PT & (controls)s & $36(15 \cdot 5)$ & $26(14)$ & $30(15.5)$ \\
APPT & (controls)s & $51(45)$ & $61(41)$ & $52(37)$ \\
Serum fibrinogen & $2-4 \mathrm{~g} / 1$ & 0.7 & 0.9 & $1 \cdot 7$ \\
FDP-D-Dimers & $<0 \cdot 25 \mathrm{mg} / 1$ & $>4$ & $>4$ & $>4$ \\
RCF & & Yes & Yes & Yes
\end{tabular}

PT prothrombin time; APTT activated partial thromboplastin time; FDP-D-Dimers fibrinogen degradation product-D-Dimers; RCF fragmented red cells in peripheral blood.

\section{Case reports \\ CASE 1}

A 78 year old woman was admitted with a one week history of breathlessness, productive cough, central chest pain, easy bruising and jaundice. Her medical history included cholecystectomy, myocardial infarction 12 months previously, and diabetes mellitus that had not been treated with insulin. On examination she was centrally cyanosed, hypotensive, and had biventricular cardiac failure, purpura, and bruising.

Haematological investigations confirmed the diagnosis of DIC (table). Biochemical investigations were as follows: plasma sodium $134 \mathrm{mmol} / 1$, potassium $5.6 \mathrm{mmol} / 1$, urea $21.3 \mathrm{mmol} / \mathrm{l}$, creatinine $140 \mu \mathrm{mol} / \mathrm{l}$, glucose $6.5 \mathrm{mmol} / \mathrm{l}$, bilirubin $30 \mu \mathrm{mol} / \mathrm{l}$, amylase $276 \mathrm{IU} / 1$, and creatine kinase $180 \mathrm{IU} / 1$. A chest radiograph showed cardiomegaly with shadowing of the middle and lower zones of the right lung. The electrocardiogram showed right bundle branch block and lateral ischaemia. She died 10 days after admission to hospital. Necropsy showed pulmonary embolism and infarction of the middle and lower lobes of the right lung.

CASE 2

A previously fit 85 year old woman presented with a two week history of breathlessness and left sided pleuritic chest pain, and a five day history of haemoptysis. Examination showed hypotension with severe cardiac failure. Two days later she developed purpura and extensive bruising around venepuncture sites. DIC was confirmed (table). The electrocardiogram showed an extensive anterior myocardial infarction and the chest radiograph confirmed left ventricular failure. A ventilation and perfusion lung scan showed a matched defect at the right lung base, not thought typical of pulmonary embolism. Twenty four hours later she was jaundiced; bilirubin $51 \mu \mathrm{mol} / 1$, creatine kinase $113 \mathrm{IU} / 1$, aspartate transaminase (AST) $2580 \mathrm{IU} / 1$ and alanine transaminase (ALT) $2220 \mathrm{IU} / 1$. An abdominal ultrasound scan showed only features of congestive cardiac failure. She died nine days after admission. Necropsy showed bilateral pulmonary emboli with infarction of the lower lobes of both lungs. There was recent thrombosis of the anterior descending branch of the left coronary artery with an extensive infarct of the anterior wall of the left ventricle covered by mural thrombus.

\section{CASE 3}

A 75 year old woman was admitted with a one month history of increasing dyspnoea. She had no clinically important medical history. She was hypothermic (core temperature $32^{\circ} \mathrm{C}$ ), hypotensive, and had purpura and severe biventricular cardiac failure.

The haemotological results were compatible with DIC (table). Biochemical investigations: plasma sodium $135 \mathrm{mmol} / \mathrm{l}$, potassium $5.3 \mathrm{mmol} / 1$, urea $17.4 \mathrm{mmol} / 1$, creatinine $203 \mu \mathrm{mol} / 1$, bilirubin $125 \mu \mathrm{mol} / 1$, creatine kinase $1781 \mathrm{IU} / 1$, AST $221 \mathrm{IU} / 1$ and ALT $149 \mathrm{IU} / 1$. The chest radiograph showed cardiomegaly, collapse of the lower lobe of the right lung, and consolidation of the lower lobe of the left lung. The electrocardiogram suggested an old anterior myocardial infarction. A ventilation and perfusion lung scan showed decreased perfusion at the left lung base, compatible with pulmonary embolism. Intravenous heparin was started but was discontinued the next day when melaena developed. She died three days after admission to hospital. Necropsy confirmed the presence of deep vein thrombosis and pulmonary embolism. There was a large left ventricular aneurysm due to an old infarct, which contained thrombus.

\section{Discussion}

DIC may be induced by factors released from hypoxic or infarcted tissue, ${ }^{1}$ but myocardial infarction and pulmonary embolism or infarction have rarely been reported as causes. Subclinical changes in circulating clotting factors occur in pulmonary embolism and to a lesser extent in deep vein thrombosis, but do not usually fulfil the definition criteria for DIC. ${ }^{2}$ These changes may be due to clotting factor consumption by the pulmonary thrombosis, ${ }^{3}$ or thromboplastin release from infarcted tissue. ${ }^{1}$ If sufficient thromboplastin is released by a large pulmonary infarct, then the coagulation abnormality can progress from symptomless haematological change to clinical DIC. The pulmonary infarcts in cases 1 and 2 were extensive, a feature they share with previous examples of DIC attributable to pulmonary infarcts. ${ }^{4}$

Abnormalities in circulating clotting factors are also seen in myocardial infarctions. Myocardium contains less thromboplastic activity than lung, however, ${ }^{6}$ and quantitatively less tissue is infarcted, thus thromboplastin release secondary to myocardial infarction may be insufficient to trigger DIC. An alternative mechanism exists whereby myocardial infarction might lead to DIC; thrombosis within a ventricular aneurysm has been associated with $\mathrm{DIC}^{7}$; both cases 2 and 3 had a large ventricular aneurysm filled with thrombus.

We believe these three cases are examples of pulmonary and myocardial infarction causing rather than complicating DIC; no other cause could be found at necropsy. Review of cases 1 and 2 after necropsy suggests that the clinical presentation was due to a combination of pulmonary embolism and infarction. In case 3 the presentation was with cardiac failure, but 
consumption of clotting factors by the large intracardiac thrombus precipitated DIC. ${ }^{7}$

The use of heparin in DIC is controversial. Stahl et al reported the successful treatment of six cases of mild consumption coagulopathy (normal or near normal clotting) due to occult pulmonary embolism, with full heparinisation. ${ }^{8}$ In contrast, Mant and King reviewed 47 patients with severe DIC $^{9}: 12$ patients had received heparin, in seven bleeding worsened, and in only one was any benefit seen.

Identification and treatment of the precipitating cause is essential in the management of DIC, but in this series the underlying cause of the DIC was only apparent at necropsy. Case 2 had a matched defect on the ventilation and perfusion lung scan which was not thought to represent pulmonary embolic disease. This investigation is neither sensitive nor specific for pulmonary embolism, however, ${ }^{10}$ and while mismatched defects are compatible with pulmonary embolism, pulmonary infarction may cause a matched defect. These cases highlight the need for early diagnosis and treatment of pulmonary embolism before the irreversible stage of severe DIC is reached. At this stage coagulation with heparin is unlikely to be of benefit. A high degree of clinical suspicion is of particular importance in the elderly, in whom clinical presentation may be atypical.

We thank Dr J Y in for advice regarding the management of these cases.

1 Preston FE. Disseminated intravascular coagulation. $\mathrm{Br} J$ Hosp Med 1982;28:129-37.

2 Ruckley CV, Das PC, Leitch AG, et al. Serum fibrin/ fibrinogen degradation products associated with postoperative pulmonary embolus and venous thrombosis. $\mathrm{Br}$ operative pulmonary
Med $J 1970 ; 4: 395-8$.

3 Cade J, Hirsh J, Regoeczi E. Mechanisms for elevated fibrin/ fibrinogen degradation products in acute experimental pulmonary embolism. Blood 1975;45:563-8.

4 McKay DG, Franciosi R, Zeller J. Pulmonary embolism and disseminated intravascular coagulation. Am J Cardiol 1967;20:374-80.

5 Okuno T, Nelson C. Value of determination of serum fibrinfibrinogen degradation products in acute myocardial infarction. Am J Clin Pathol 1974;61:155-9.

6 Astrup T. Assay and content of tissue thromboplastin in different organs. Thromb Diath Haem 1965;14:401-16.

7 Sculier JP, Coune A, Vandenbossche JL, Muller C. Intracardiac thrombosis associated with an acute consumintracardiac thrombosis associated with an acutect

8 Stahl RL, Javid JP, Lackner H. Unrecognised pulmonary embolism presenting as disseminated intravascular coagulation. Am J Med 1984;76:772-8.

9 Mant MJ, King EG. Severe, acute disseminated intravascular coagulation. Am J Med 1979;67:557-63.

10 Caracci BF, Rumbolo PM, Mainini S, Walker III HSJ, Peterson GJ. How accurate are ventilation-perfusion lung scans for pulmonary embolism? Am J Surg 1988;156: 477-80. 